\title{
The growth of Gardnerella vaginalis and Lactobacillus acidophilus in Sorbarod biofilms
}

\author{
F. W. MULI and J. K. STRUTHERS \\ Department of Medical Microbiology, Clinical Sciences Building, Manchester Royal Infirmary and the \\ University of Manchester, Oxford Road, Manchester M13 9WL
}

\begin{abstract}
Sorbarod biofilms were investigated for their suitability in establishing continuous culture biofilms for the study of bacterial vaginosis. Two important organisms in the condition, Gardnerella vaginalis and Lactobacillus acidophilus, were studied. In contrast to growth in broth culture, both organisms were maintained for at least $96 \mathrm{~h}$ in a steady state on the biofilms. With $G$. vaginalis, the haemolytic activity was consistently maintained in the biofilms in contrast to short-term activity in broth culture which matched the bacterial titre. The simple Sorbarod system appears to be suitable for studying the growth conditions of bacteria in continuous culture and has potential for investigating interactions between micro-organisms.
\end{abstract}

\section{Introduction}

Bacterial vaginosis is a condition that is still poorly understood [1]. Apart from the importance of the condition as a cause of vaginal discharge, its significance has been emphasised recently by work that has shown an association between bacterial vaginosis and premature delivery of low-birth-weight infants [2,3]. Treatment of 'at risk' mothers with metronidazole and erythromycin resulted in a reduction in the rates of premature delivery [2]. The implications of this finding for possibly reducing the incidence of premature infants and the effect on admissions to neonatal medical units could be profound.

The normal vaginal flora is dominated by lactobacilli such as Lactobacillus acidophilus. In bacterial vaginosis this is replaced with a mixed flora of aerobic, facultative and anaerobic micro-organisms including Gardnerella vaginalis, Mobiluncus spp. and Mycoplasma hominis $[4,5]$. G. vaginalis is often identified in clinical specimens by the presence of 'clue' cells, consisting of vaginal epithelial cells to which these bacteria adhere in large numbers [1]. G. vaginalis is haemolytic on human blood agar; however, the role of this in the pathogenesis of the condition remains unclear [4].

The details of the physiological and pathological mechanisms that underly bacterial vaginosis remain

Received 15 April 1997; revised version accepted 3 Sept. 1997.

Corresponding author: Dr J. K. Struthers. largely unresolved, reflecting in part the lack of a suitable in-vitro model. A recent publication described a system that could have potential for studying growth of individual organisms as well as the interaction of a wide range of micro-organisms grown together in biofilms. Hodgson et al. [6] used Sorbarods, consisting of a cellulose cylinder in a paper sleeve, to show that both Staphylococcus aureus and Pseudomonas aeruginosa could be maintained for days at high concentrations in what was essentially a simple continuous culture biofilm system. The work presented here investigated the appropriateness of this system as a model for vaginosis by determining the growth characteristics of $G$. vaginalis and $L$. acidophilus, separately and together, on Sorbarods.

\section{Materials and methods}

\section{Bacteria and media}

G. vaginalis ATCC 14018 and L. acidophilus ATCC 832 were used as the test strains. Columbia Agar (Oxoid) containing defibrinated horse blood 7\% was usually used for subculture and titration. When mixtures of $G$. vaginalis and $L$. acidophilus were grown together on biofilms, de Man, Rogosa, Sharpe (MRS) selective agar (Oxoid) was also used to determine the number of lactobacilli present, as the titres of this organism were generally $1-2 \log _{10}$ values lower than $G$. vaginalis. All plates were incubated in an atmosphere of air with $\mathrm{CO}_{2} 6 \%$ at $37^{\circ} \mathrm{C}$.

When micro-organisms were grown in broth culture, 50$100-\mathrm{ml}$ volumes of broth were inoculated and incubated 
at $37^{\circ} \mathrm{C}$ on a rotating table set at a speed at $150 \mathrm{rpm}$. For optimum growth of $G$. vaginalis and $L$. acidophilus, Brain Heart Infusion (BHI) Broth (Oxoid) and MRS Broth (Oxoid) were used for the respective species.

\section{Sorbarod biofilms}

Sorbarod biofilms were prepared by a modification of the method of Hodgson et al. [6]. Individual $10 \mathrm{~mm}$ diameter by $20 \mathrm{~mm}$ length cellulose Sorbarods (Ilacon Ltd, Kent) were placed in a $10-\mathrm{cm}$ length of silicone tubing. These were then connected by $0.8-\mathrm{mm}$ internal bore tubing to a supply of broth. The effluent end of the biofilm apparatus was connected via plastic adaptors to replaceable sterile $150-\mathrm{ml}$ glass bottles, enabling collection of effluent fluid at any time point in the experiments. The entire apparatus, consisting of a supply bottle of BHI or MRS broth, connecting tubing, Sorbarod and effluent bottle was then autoclaved as a single unit. Individual Sorbarods were prewetted with $3 \mathrm{ml}$ of BHI or MRS broth and inoculated with $3 \mathrm{ml}$ of an overnight broth culture of the relevant organism(s) either separately or in combination. The broth 'feed' was delivered to each Sorbarod by means of a Watson Marlow 12 channel 205U peristaltic pump at a flow rate of $0.1 \mathrm{ml} / \mathrm{min}$. The whole apparatus, consisting of up to 12 Sorbarods was incubated at $37^{\circ} \mathrm{C}$. At each assay time point, effluent was collected in a sterile container for $10 \mathrm{~min}$ so that the titre of planktonic bacteria could be determined. Individual biofilms were then harvested in $5 \mathrm{ml}$ of broth, vortex mixed to disintegrate the cellulose matrix, and $100 \mu \mathrm{l}$ of the bacterial suspension were removed for titration, which was done in triplicate by surface viable count [7].

\section{G. vaginalis haemolytic activity}

The haemolytic activity of $G$. vaginalis was determined with group $\mathrm{O}$ human red blood cells washed four times in phosphate-buffered saline (PBS, $\mathrm{pH}$ 7.2) and then resuspended in BHI. The haematocrit was determined and this value was used to prepare red blood cell (RBC) suspensions with concentrations of $0.6,1.25,5$ and $10 \% \mathrm{v} / \mathrm{v}$. At the indicated times, samples of broth culture were removed and the supernate was obtained by centrifugation at $3000 \mathrm{rpm}$ for $10 \mathrm{~min}$. After vortex mixing and titration of a biofilm, the remainder of the vortexate was centrifuged at $3000 \mathrm{rpm}$ for $10 \mathrm{~min}$ to obtain a supernatant fraction of the vortexate. Equal volumes $(150 \mu \mathrm{l})$ of broth or biofilm supernate and RBC preparations were mixed in 96-well microtitration plates (Sterilin) and incubated for $30 \mathrm{~min}$ at $37^{\circ} \mathrm{C}$. The plates were then centrifuged at $2000 \mathrm{rpm}$ for $10 \mathrm{~min}$ in a Sigma SK10 centrifuge to remove intact $\mathrm{RBC}$ and $150 \mu \mathrm{l}$ of the supernate were removed and diluted in two-fold steps in BHI. Plates were scanned at $415 \mu \mathrm{m}$ in a Multiscan MCC/340 MKII plate reader and the percentage haemolysis index for each RBC concentration was determined by the following formula:

\section{Haemolysis index (\%)}

$$
=\frac{\mathrm{OD}_{415} \times \text { dilution factor of test } \mathrm{RBC}}{\mathrm{OD}_{415} \times \text { dilution factor of control } \mathrm{RBC}}
$$

Completely lysed control RBC were obtained by making equivalent $\mathrm{RBC}$ suspensions $3 \% \mathrm{v} / \mathrm{v}$ with a stock solution of saponin (BDH) $10 \% \mathrm{w} / \mathrm{v}$. These preparations were processed in the same way as test specimens.

\section{Results}

Typical growth curves of $G$. vaginalis in broth culture and on the Sorbarod biofilms with BHI are shown in Fig. 1. In broth, $G$. vaginalis reached a maximum titre within $24-48 \mathrm{~h}$ and then entered the stationary and decline phases. In biofilms, titres in excess of $10^{9} \mathrm{cfu} / \mathrm{ml}$ were achieved within $24 \mathrm{~h}$ and these were maintained for at least $96 \mathrm{~h}$. The concentration of bacteria in the effluent was in the order of $2 \log _{10}$ values lower than that obtained from the Sorbarod biofilms. With this strain, it was important that Sorbarods were inoculated with broth cultures of titre $>10^{6} \mathrm{cfu} / \mathrm{ml}$, as lower inocula failed to establish themselves on the Sorbarods.

Growth of L. acidophilus in broth and biofilm with MRS broth is shown in Fig. 2. The maximum titre in broth was obtained at $24-48 \mathrm{~h}$. In biofilm, titres remained stable over the $96-\mathrm{h}$ period; the titres obtained in the biofilms were regularly in excess of $10^{9} \mathrm{cfu} / \mathrm{ml}$. Growth of L. acidophilus in BHI broth and biofilm is shown in Fig. 3. These titres were several $\log _{10}$ values lower than $L$. acidophilus grown in MRS, but are similar to those obtained by other workers studying lactobacilli [8].

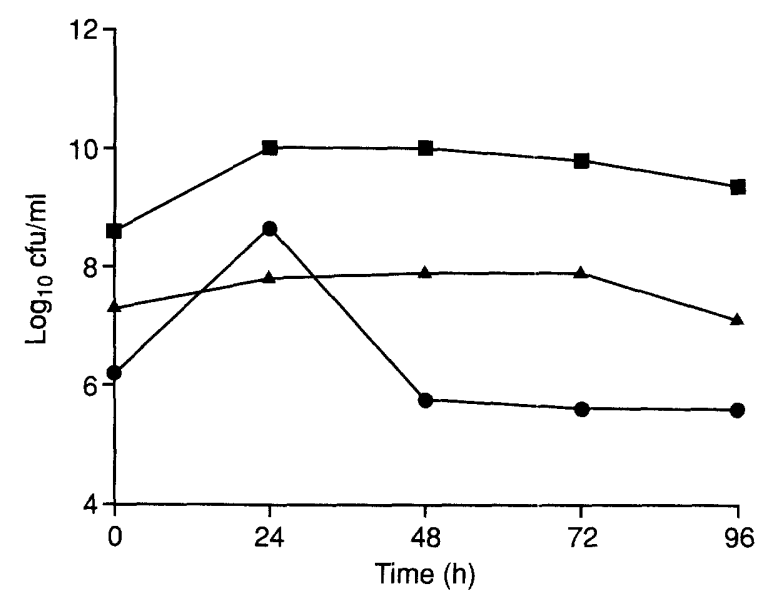

Fig. 1. Growth of $G$. vaginalis in broth culture (-) and in Sorbarod biofilm with BHI broth. At the indicated times biofilm effluent was collected for $10 \mathrm{~min}$ to determine the titre of planktonic bacteria $(\boldsymbol{\Delta}-\mathbf{\Delta})$. An individual biofilm was then harvested and vortex mixed to determine the biofilm titre 


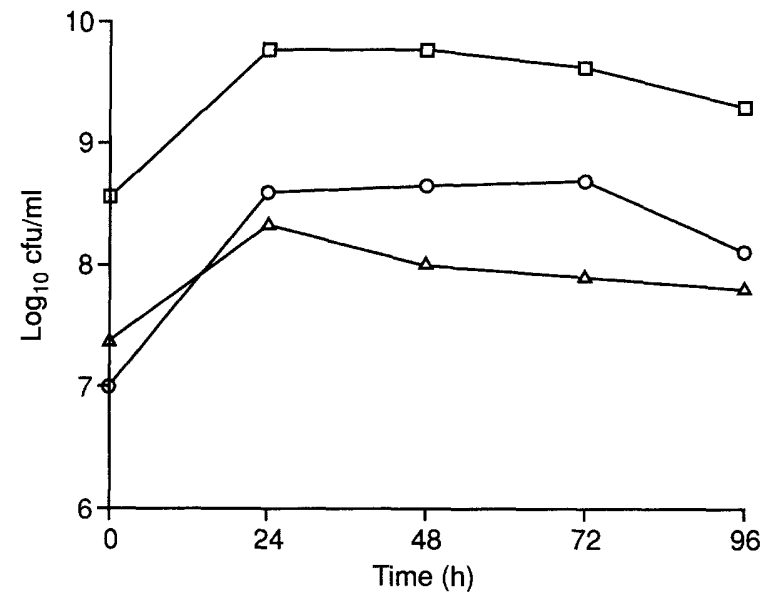

Fig. 2. Growth of L. acidophilus in broth culture (O-O) and in Sorbarod biofilm with MRS broth. At the indicated times biofilm effluent was collected for $10 \mathrm{~min}$ to determine the titre of planktonic bacteria $(\triangle$ $\triangle)$. An individual biofilm was then harvested and vortex mixed to determine the biofilm titre ( $\square-\square)$.

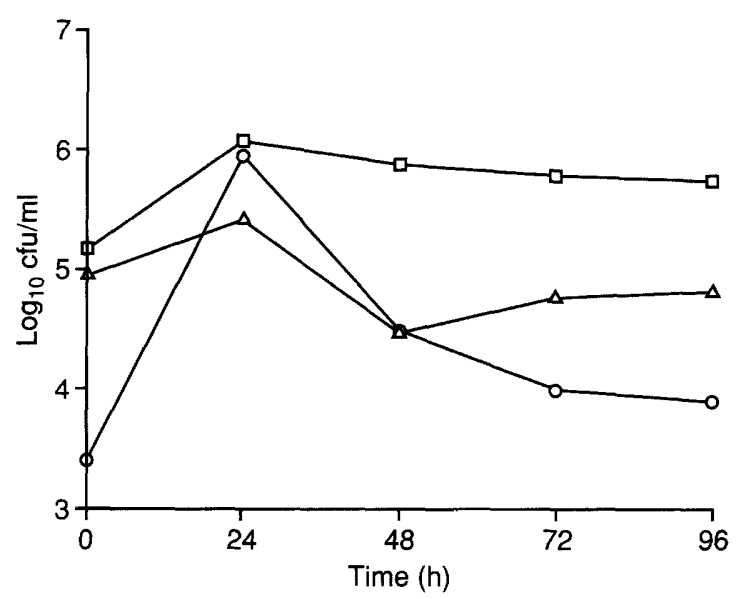

Fig. 3. Growth of L. acidophilus in broth culture $(\mathrm{O}-\mathrm{O})$ and in Sorbarod biofilm with BHI broth. At the indicated times effluent was collected for $10 \mathrm{~min}$ to determine the titre of planktonic bacteria $(\triangle-\triangle)$. An individual biofilm was then harvested and vortex mixed to determine the biofilm titre $(\square-\square)$.

The growth pattern of both organisms in the same Sorbarod with BHI broth is shown in Fig. 4. The lactobacillus pattern was similar to that of the organism grown alone on the BHI-fed biofilm. With $G$. vaginalis there was a repeatable drop in titre at 24 and $48 \mathrm{~h}$ with recovery to high levels from $72 \mathrm{~h}$ onwards. Thus, the relative ratio of $G$. vaginalis and L. acidophilus varied from $10: 1$ to $100: 1$ at 24 and $48 \mathrm{~h}$ to $>1000: 1$ from $72 \mathrm{~h}$ onwards.

The haemolytic activity in the supernate of $G$. vaginalis grown in broth culture is shown in Fig. 5. Haemolytic activity was maximal at $24 \mathrm{~h}$, corresponding with the maximum titre in the broth; there was minimal activity at $96 \mathrm{~h}$. The result of the assay for haemolytic activity in biofilms is shown in Fig. 6. For

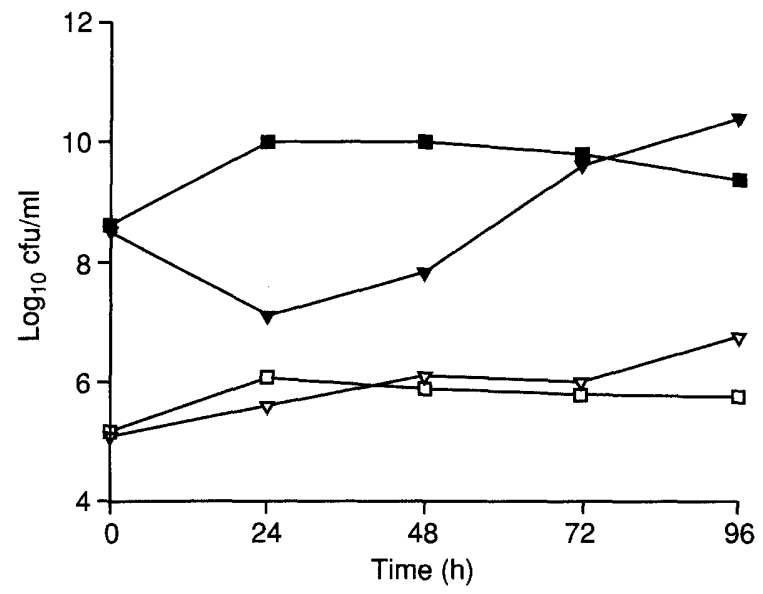

Fig. 4. Growth of G. vaginalis ( $\mathbf{\square}-\mathbf{0}$ ) and L. acidophilus ( $\square-\square)$ grown separately in Sorbarod biofilms with BHI broth, compared to growth together in the same biofilm (G. vaginalis: $\nabla-\nabla ;$ L. acidophilus: $\nabla-\nabla)$.
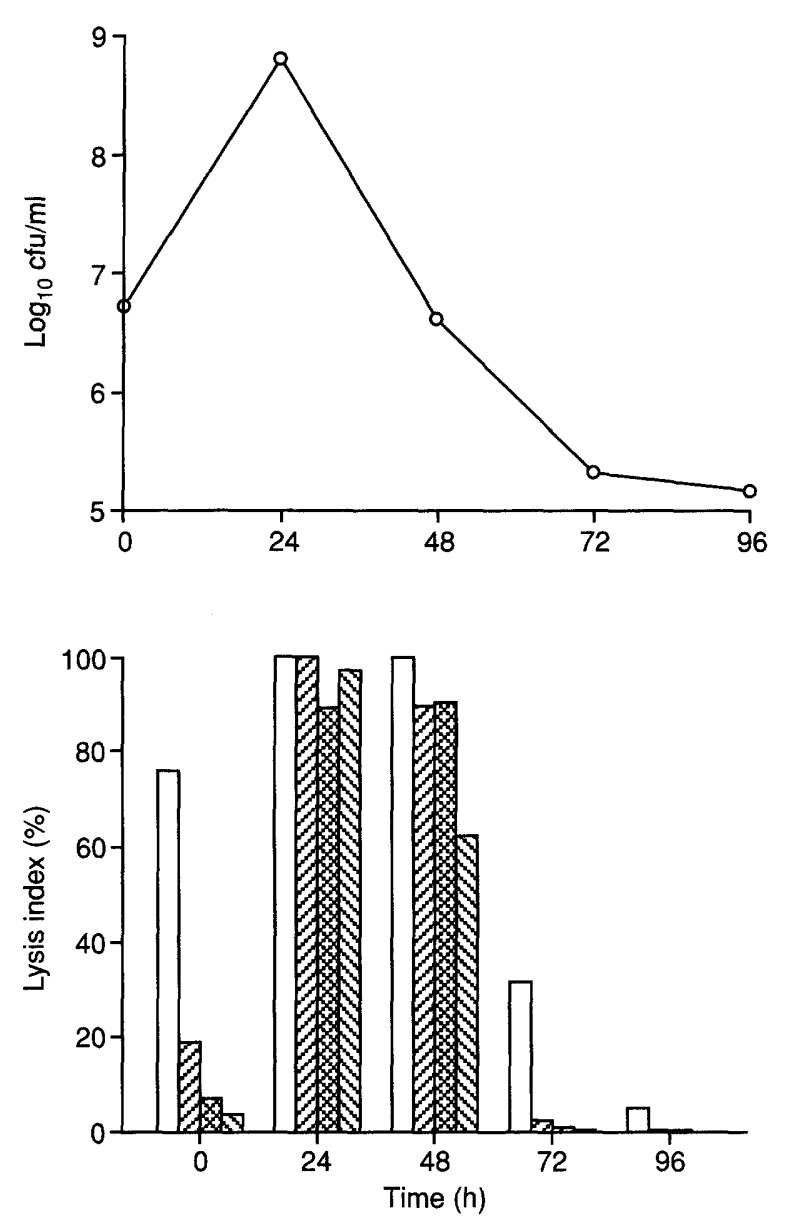

Fig. 5. Growth curve of $G$. vaginalis in broth culture and the associated percentage haemolytic index. At each time point a sample was collected for titration and the supernate was assayed for haemolytic activity. The final concentrations of human red blood cells used at each time point were: $0.3 \square, 0.6 \square, 2.5$ and $5 \% \mathrm{v} / \mathrm{v}$.

the $0.3-2.5 \%$ RBC (final) concentrations, the haemolytic index ranged from 92 to $100 \%$ from $24 \mathrm{~h}$ onwards. 

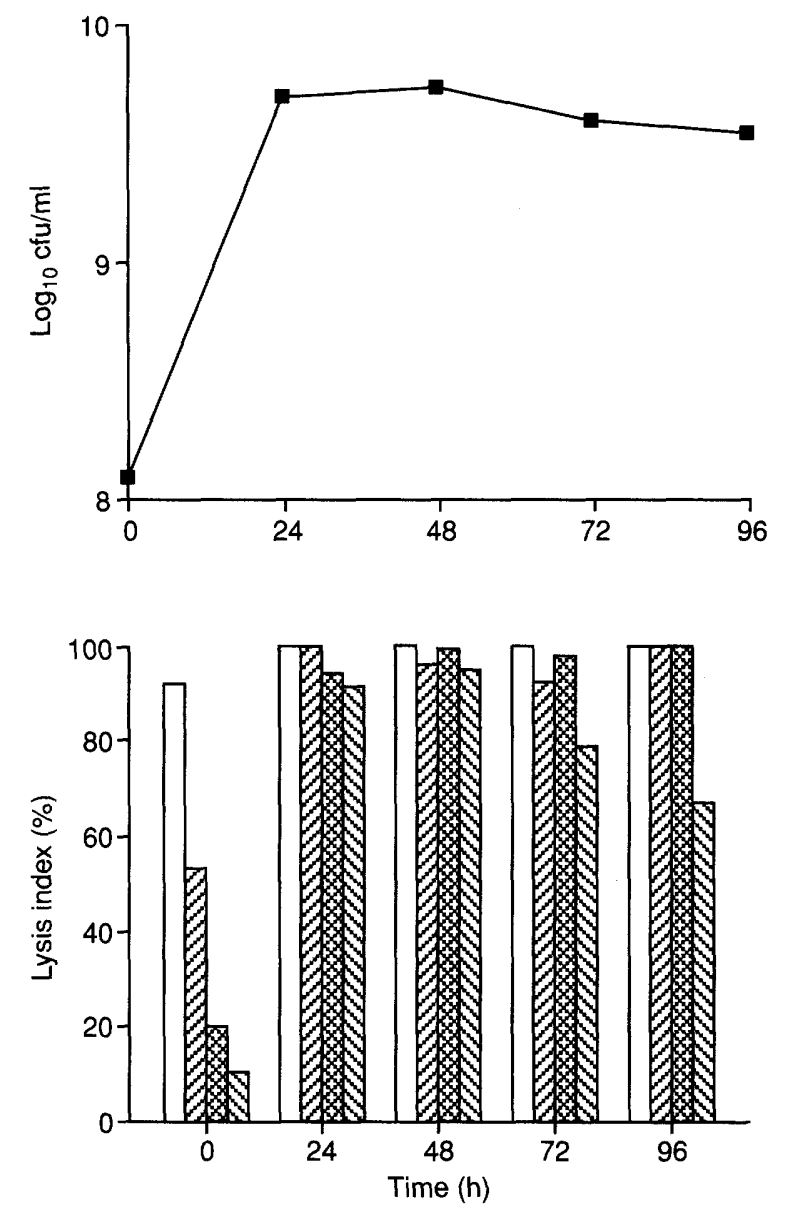

Fig. 6. Growth of $G$. vaginalis in biofilms and the associated percentage haemolytic index. At each time point a biofilm was harvested and vortex mixed. A sample was collected for titration. The remainder of the 'vortexate' was then centrifuged and the haemolytic activity was determined. The final concentrations of the human red blood cells used at each time point were: 0.3 $\square, 0.6 \square, 2.5$ and $5 \% \mathrm{v} / \mathrm{v}$.

\section{Discussion}

Bacterial vaginosis is a significant cause of a vaginal discharge $[1,4]$. Furthermore, the recent work clarifying the role of the condition in premature delivery makes this an important area for investigation $[2,3]$. The aim of the present work was to characterise the growth of $G$. vaginalis and $L$. acidophilus by the biofilm system of Hodgson et al. [6]. These workers showed that when $S$. aureus and P. aeruginosa were grown alone, both achieved high concentrations over a period of $96 \mathrm{~h}$. G. vaginalis reached steady state within $24 \mathrm{~h}$ on the biofilms and maintained this for at least $96 \mathrm{~h}$. Thin sections of these biofilms show numerous microcolonies of the organism (30-50 $\mu \mathrm{m}$ in diameter) in addition to individual organisms adherent to the cellulose matrix (F. W. Muli, unpublished observations). When the G. vaginalis inoculum was diluted to $\leqslant 10^{6} \mathrm{cfu} / \mathrm{ml}$, the organism failed to establish itself on the biofilm, indicating a critical inoculum effect. $L$. acidophilus likewise achieved a high steady state concentration when grown in MRS broth. The growth of the two organisms in the same biofilms with BHI is shown in Fig. 4. While the titre of L. acidophilus remained constant, in the order of $10^{6} \mathrm{cfu} / \mathrm{ml}$, that of G. vaginalis consistently fell between 24 and $48 \mathrm{~h}$. Whether this relates to antibiosis between lactobacilli and G. vaginalis, as noted by other workers [9], remains to be elucidated.

While extensive knowledge of individual species such as $G$. vaginalis and the lactobacilli has been obtained, studies on the interaction of these bacteria have largely been limited to analysis of the organisms in clinical isolates $[4,5,9,10]$. The ecological change from the normal vaginal flora to one that characterises bacterial vaginosis remains to be defined. It has been pointed out that bacteria growing in vivo probably exist within biofilms [11]. While the main emphasis of the study of bacteria within biofilms in vitro has related to the effect of antibiotics in such experimental conditions, important factors affecting bacterial growth such as the availability of nutrients have been highlighted [12]. The system reported here with BHI indicates that the interactions between the bacteria can be studied between 24 and $48 \mathrm{~h}$ when there is a reasonable ratio of $G$. vaginalis to lactobacilli of between $10: 1$ and 100:1. Other general and selective media were investigated for the growth of both organisms. These included preparation of MRS broth from its individual constituents and selectively adding the lactobacillus growth promotors sorbitan mono-oleate (Sigma) and sodium acetate [13]. While MRS broth without these compounds grew G. vaginalis to titres $>10^{8} \mathrm{cfu} / \mathrm{ml}$, the absence of sorbitan mono-oleate gave poor growth of the lactobacillus with titres $<10^{5} \mathrm{cfu} / \mathrm{ml}$. Both sorbitan mono-oleate and sodium acetate repressed the growth of G. vaginalis. Similarly, BHI with sorbitan mono-oleate 0.01 or $0.001 \% \mathrm{v} / \mathrm{v}$ supported lactobacilli to titres $>10^{7} \mathrm{cfu} / \mathrm{ml}$, but inhibited $G$. vaginalis. Concentrations of sorbitan mono-oleate $<0.001 \%$ in BHI failed to support the growth of lactobacilli. As interaction between the micro-organisms is probably important in the pathogenesis of bacterial vaginosis, we are currently reviewing the semi-defined medium developed for G. vaginalis in 1961 [14] with a view to developing a defined medium to grow both organisms in this continuous culture biofilm system.

The fact that the system can be used to study the growth of the bacteria in steady state enabled a preliminary assessment of the haemolytic activity of $G$. vaginalis. The haemolysin produced has been shown by Cauci et al. [15] to be heat liable. This was confirmed by determining the activity in broth culture over a 96-h period. A significant reduction in the haemolytic activity paralleled the titre of bacteria. In contrast to this short-lived overall activity, the activity in the biofilm remained consistently high over the 96-h study period. Therefore, this continuous culture system would be suitable for investigating the expression of this pathogenic property of G. vaginalis. 
One of the main advantages of the Sorbarod system is that environmental conditions can be changed without physical manipulation of the biofilm, by using an upstream ' $T$ ' connector. The system is labour intensive but it appears to be suitable for studying the interactions of bacteria in biofilms.

F.W.M. is on sabbatical leave from the Moi University, Kenya. This is financed by a Post Graduate grant from the World Bank, Washington, USA.

\section{References}

1. Easmon CSF, Hay PE, Ison CA. Bacterial vaginosis: a diagnostic approach. Genitourin Med 1992; 68: 134-138.

2. Hauth JC, Goldenberg RL, Andrews WW, DuBard MB, Copper $\mathrm{RL}$. Reduced incidence of preterm delivery with metronidazole and erythromycin in women with bacterial vaginosis. $N$ Engl J Med 1995; 333: 1732-1736.

3. Hillier SL, Nugent RP, Eschenbach DA and the Vaginal Infections and Prematurity Study Group. Association between bacterial vaginosis and preterm delivery of low-birth-weight infant. $N$ Engl $J$ Med 1995; 333: 1737-1742.

4. Spiegel CA. Bacterial vaginosis. Clin Microbiol Rev 1991; 4 485-502.

5. Rosenstein IJ, Morgan DJ, Sheehan M, Lamont RF, Taylor-
Robinson D. Bacterial vaginosis in pregnancy: distribution of bacterial species in different gram-stain categories of the vaginal flora. $J$ Med Microbiol 1996; 45: 120-126.

6. Hodgson AE, Nelson SM, Brown MRW, Gilbert P. A simple in vitro model for growth control of bacterial biofilms. $J$ Appl Bacteriol 1995; 79: 87-93.

7. Miles AA, Misra SS, Irwin JD. The estimation of the bacteriocidal power of the blood. J Hygiene 1938; 38: 732-749.

8. Jones JW, Paull SN. Effect of biofilm culture on antibiotic susceptibility of lactobacilli causing endocarditis. $J$ Infect 1995 ; 31: $80-81$.

9. Nagy E, Petterson M, Mårdh P-A. Antibiosis between bacteria isolated from the vagina of women with and without signs of bacterial vaginosis. APMIS 1991; 99: 739-744.

10. Holst E. Reservoir of four organisms associated with bacterial vaginosis suggests lack of sexual transmission. $J$ Clin Microbiol 1990; 28: 2035-2039.

11. Gander S. Bacterial biofilms: resistance to antimicrobial agents. $J$ Antimicrob Chemother 1996; 37: 1047-1050.

12. Nichols WW. Biofilms, antibiotics and penetration. Rev Med Microbiol 1991; 2: 177-181.

13. Sharpe ME, Fryer TF, Smith DG. Identification of the lactic acid bacteria. In: Gibbs BM, Skinner FA (eds) Identification methods for microbiologists, part A. New York, Academic Press. 1966: 65-79.

14. Dunkelberg WE, McVeigh I. Growth requirements of Haemophilus vaginalis. Antonie van Leeuwenhoek 1969; 35: 129-145.

15. Cauci S, Monte R, Ropele $\mathrm{M}$ et al. Pore-forming and haemolytic properties of the Gardnerella vaginalis cytolysin. Mol Microbiol 1993; 9: 1143-1155. 